\title{
Antifungal properties of volatile organic compounds produced by Daldinia eschscholtzii MFLUCC 19-0493 isolated from Barleria prionitis leaves against Colletotrichum acutatum and its post-harvest infections on strawberry fruits
}

\author{
Sarunpron Khruengsai ${ }^{1}$, Patcharee Pripdeevech ${ }^{\text {Corresp., } 1,2}$, Chutima Tanapichatsakul ${ }^{1}$, Chanin Srisuwannapa ${ }^{1}$, Priya \\ Esilda D'Souza ${ }^{3}$, Parinya Panuwet ${ }^{\text {Corresp. } 3}$ \\ ${ }^{1}$ School of Science, Mae Fah Luang University, Muang, Chiang Rai, Thailand \\ 2 Center of Chemical Innovation for Sustainability (CIS), Mae Fah Luang University, Muang, Chiang Rai, Thailand \\ 3 Laboratory of Exposure Assessment and Development for Environmental Research, Gangarosa Department of Environmental Health, Rollins School of \\ Public Health, Emory University, Atlanta, Georgia, United States \\ Corresponding Authors: Patcharee Pripdeevech, Parinya Panuwet \\ Email address: patcharee.pri@mfu.ac.th, parinya.panuwet@emory.edu
}

Fungal endophytes are microorganisms living symbiotically with a host plant. They can produce volatile organic compounds (VOCs) that have antimicrobial activity. This study aimed to isolate endophytic fungi from Barleria prionitis plants grown in Thailand and to investigate the antifungal properties of their VOCs against Colletotrichum acutatum, a causal agent of anthracnose disease on post-harvest strawberry fruits. A total of 34 endophytic fungi were isolated from leaves of $B$. prionitis. The VOCs produced from each individual isolate were screened for their antifungal activity against $C$. acutatum using a dual-culture plate method. From this in vitro screening experiment, the VOCs produced by the endophytic isolate BP11 were found to have the highest inhibition percentage $(80.3 \%)$ against the mycelial growth of $C$. acutatum. The endophytic isolate BP11 was molecularly identified as Daldinia eschscholtzii MFLUCC 19-0493. This strain was then selected for an in vivo experiment. Results from the in vivo experiment indicated that the VOCs produced by $D$. eschscholtzii MFLUCC 19-0493 were able to inhibit infections by $C$. acutatum on organic fresh strawberry fruits with an inhibition percentage of $72.4 \%$. The quality of the pathogen-inoculated strawberry fruits treated with VOCs produced by $D$. eschscholtzii MFLUCC 19-0493 was evaluated. Their fruit firmness, total soluble solids, and pH were found to be similar to the untreated strawberry fruits. Solid phase microextraction-gas chromatographic-mass spectrometric analysis of the VOCs produced by D. eschscholtzii MFLUCC 19-0493 led to the detection and identification of 60 compounds. The major compounds were elemicin (23.8\%), benzaldehyde dimethyl acetal (8.5\%), ethyl sorbate (6.8\%), methyl geranate (6.5\%), trans-sabinene hydrate (5.4\%), and 3,5-dimethyl-4heptanone $(5.1 \%)$. Each was tested for its antifungal activity against $C$. acutatum using the 
in vitro assay. While all these selected VOCs showed varying degrees of antifungal activity, elemicin was found to possess the strongest antifungal activity. This work suggests that $D$. eschscholtzii MFLUCC 19-0493 could be a promising natural preservative for controlling $C$. acutatum associated anthracnose disease in strawberry fruits during the post-harvest period. 
1 Antifungal properties of volatile organic compounds

2 produced by Daldinia eschscholtzii MFLUCC 19-0493

3 isolated from Barleria prionitis leaves against

4 Colletotrichum acutatum and its post-harvest

5 infections on strawberry fruits

6

7

8

9

10

11

12

13

14

15

16

17

18

19

20

21

22

23

24

25

26

27

28

29

30

31

32

33

34

35

36

37

Sarunpron Khruengsai ${ }^{1}$, Patcharee Pripdeevech ${ }^{1,2}$, Chutima Tanapichatsakul ${ }^{1}$, Chanin

Srisuwannapa ${ }^{1}$, Priya Esilda D'Souza ${ }^{3}$, Parinya Panuwet ${ }^{3}$

${ }^{1}$ School of Science, Mae Fah Luang University, Chiang Rai 57100, Thailand

${ }^{2}$ Center of Chemical Innovation for Sustainability (CIS), Mae Fah Luang University, Chiang Rai, 57100, Thailand

${ }^{3}$ Laboratory of Exposure Assessment and Development for Environmental Health Research, Gangarosa Department of Environmental Health, Rollins School of Public Health, Emory

University, Atlanta, Georgia, 30322, USA

Corresponding Author:

Patcharee Pripdeevech ${ }^{1,2}$

School of Science, Mae Fah Luang University, Chiang Rai 57100, Thailand

Center of Chemical Innovation for Sustainability (CIS), Mae Fah Luang University, Chiang Rai, 57100, Thailand

Email address: patcharee.pri@mfu.ac.th

Parinya Panuwet ${ }^{3}$

Laboratory of Exposure Assessment and Development for Environmental Research, Gangarosa

Department of Environmental Health, Rollins School of Public Health, Emory University,

Atlanta, Georgia, 30322, USA

Email address: ppanuwet@emory.edu 
38

39

40

41

42

43

44

45

46

47

48

49

50

51

52

53

54

55

56

57

58

59

60

61

62

63

64

65

66

67

68

69

70

71

72

73

74

75

76

77

\section{Abstract}

Fungal endophytes are microorganisms living symbiotically with a host plant. They can produce volatile organic compounds (VOCs) that have antimicrobial activity. This study aimed to isolate endophytic fungi from Barleria prionitis plants grown in Thailand and to investigate the antifungal properties of their VOCs against Colletotrichum acutatum, a causal agent of anthracnose disease on post-harvest strawberry fruits. A total of 34 endophytic fungi were isolated from leaves of $B$. prionitis. The VOCs produced from each individual isolate were screened for their antifungal activity against $C$. acutatum using a dual-culture plate method. From this in vitro screening experiment, the VOCs produced by the endophytic isolate BP11 were found to have the highest inhibition percentage $(80.3 \%)$ against the mycelial growth of $C$. acutatum. The endophytic isolate BP11 was molecularly identified as Daldinia eschscholtzii MFLUCC 19-0493. This strain was then selected for an in vivo experiment. Results from the in vivo experiment indicated that the VOCs produced by D. eschscholtzii MFLUCC 19-0493 were able to inhibit infections by $C$. acutatum on organic fresh strawberry fruits with an inhibition percentage of $72.4 \%$. The quality of the pathogen-inoculated strawberry fruits treated with VOCs produced by $D$. eschscholtzii MFLUCC 19-0493 was evaluated. Their fruit firmness, total soluble solids, and $\mathrm{pH}$ were found to be similar to the untreated strawberry fruits. Solid phase microextraction-gas chromatographic-mass spectrometric analysis of the VOCs produced by $D$. eschscholtzii MFLUCC 19-0493 led to the detection and identification of 60 compounds. The major compounds were elemicin (23.8\%), benzaldehyde dimethyl acetal $(8.5 \%)$, ethyl sorbate $(6.8 \%)$, methyl geranate $(6.5 \%)$, trans-sabinene hydrate $(5.4 \%)$, and 3,5-dimethyl-4-heptanone (5.1\%). Each was tested for its antifungal activity against $C$. acutatum using the in vitro assay. While all these selected VOCs showed varying degrees of antifungal activity, elemicin was found to possess the strongest antifungal activity. This work suggests that $D$. eschscholtzii MFLUCC 19-0493 could be a promising natural preservative for controlling C. acutatum associated anthracnose disease in strawberry fruits during the post-harvest period.

\section{Introduction}

Strawberry (Fragaria $\times$ ananassa Duch.) is a common edible fruit of great economic importance. It can be cultivated throughout the world, but mostly in areas with temperatures ranging from 15 to $25^{\circ} \mathrm{C}$ (Natsheh et al., 2015). Strawberries are a good source of vitamin $\mathrm{C}$, folate, and phenolic compounds. Antioxidant compounds, such as anthocyanin and ellagitannins, are also detected in strawberry fruits in high amounts (Giampieri et al., 2012). In Thailand, strawberries can only be cultivated in the few northern and northeastern provinces that are at the appropriate elevations and have suitable ambient temperatures, such as Chiang Mai, Chiang Rai, and Loei (Riyaphan et al., 2004). Due to high demand for domestic consumption, strawberries have been imported from several countries to Thailand with a total market value of hundreds of millions of baht per year (Pipattanawong, 2015).

The quality of strawberries is determined by their firmness, taste, and flavor, as well as their color, shape, and the presence or absence of defects (Kader, 1991). However, infections by 
78 pathogens during the cultivation and post-harvest periods can drastically affect this quality 79 (Sistrunk \& Morris, 1983). The pathogen that usually causes significant fruit rot disease 80 (anthracnose disease) in strawberries during the post-harvest period is the fungal pathogen 81 Colletotrichum acutatum. Infection by this pathogen contributes to significant loss in the market 82 value of fresh strawberries (Arroyo et al., 2007). Anthracnose disease also occurs in fruits such as 83 peaches, almonds, avocados, mangoes, papayas, and guavas, especially during the post-harvest

85

86

87

88

89

90

91

92

93

94

95

96

97

98

99

100

101

102

103

104

105

106

107

108

109

110

111

112

113

114

115

116

117 period (Regnier et al., 2010). Chemical fungicides (e.g., azoxystrobin, pyraclostrobin, and captan) are typically used to control anthracnose disease in strawberries during cultivation (Chalfoun et al., 2011). For post-harvest prevention of anthracnose disease, methyl bromide has been used. However, it is now being phased out in many countries because it is an ozone-depleting substance. Several other chemicals such as ethyl formate, nitric oxide, and hydrogen sulfide are now used as alternative fumigants (Kuchi \& Sharavani, 2019). The use of these chemicals, especially nitric oxide, requires a sophisticated system, which may not be accessible to small-scale farmers (Kuchi \& Sharavani, 2019). Thus, more alternative fumigation treatments capable of suppressing anthracnose disease in fresh strawberry fruits during post-harvest storage, transportation, and market periods, are needed.

Over the years, much attention has been paid to the application of biological agents to control pests and pathogen-related diseases. This is known as biocontrol (Liu et al., 2016). One biocontrol technique is the use of VOCs produced by microorganisms to inhibit the growth of and infections by pathogens on the fruit surface during the post-harvest period. This is also called postharvest biofumigation (Kaddes et al., 2019; Chen et al., 2020). Many studies have reported the successful use of VOCs produced by microorganisms, especially endophytic fungi, as biological agents to control fruit and vegetable diseases. Endophytic fungi are microorganisms asymptomatically colonizing and living in plant tissue, especially leaves, stems, and roots (Wan et al.,2008; Morath et al., 2012). Some of the endophytic fungi, such as those of the Daldinia genus, are capable of producing bioactive compounds against a variety of phytopathogens. For instance, Pandey and Banerjee (2014) reported that the endophytic fungus Daldinia bambusicola Ch4/11 produced bioactive metabolites such as linalool, benzeneethanol, 2H-1-benzopyran-2-one, pivalic acid anhydride, and 2-ethylhexanol, which were able to suppress the growth of several plant pathogens, including Colletotrichum lagenarium. Similarly, the VOCs produced by the endophytic fungus Daldinia cf. concentrica, which included 3-methyl-1-butanol, ( \pm )-2-methyl1-butanol, 4heptanone, isoamyl acetate, and trans-2-octenal, were considered to possess antifungal activities against various plant pathogenic fungi (Liarzi et al., 2016). Other fungi that produced VOCs against plant pathogens include Trichoderma (Valenzuela et al., 2015), Noduliosporium (Suwannarach et al., 2013), and Muscador albus (Mercier \& Jiménez, 2004). According to previous studies, endophytic fungi capable of producing volatile compounds that can be used for biofumigation include Streptomyces globisporus JK-1 (Droby \& Lichter, 2007; Li et al., 2010), Trichoderma (Valenzuela et al., 2015), Noduliosporium (Suwannarach et al., 2013), and Muscador albus (Mercier \& Jiménez, 2004). The use of microorganism-derived VOCs for fumigation of fresh fruits is relatively inexpensive and may require less sophisticated systems, in comparison to 
118 the use of toxic chemicals. Thus, biofumigation can likely be performed by local farmers to extend 119 the shelf-lives of their agricultural produce. It is also considered an environmentally- and user120 friendly technique. Although the use of microorganism-derived VOCs as biofumigants shows a 121 promising advantage in controlling post-harvest diseases, not many studies are available in the 122 literature that present results on the use of microorganism-derived VOCs to prevent anthracnose 123 diseases in fresh strawberry fruits (Huang et al., 2012; Chen et al., 2020; Alijani et al., 2019; Zhang 124 et al., 2015). This warrants additional research studies that may lead to the successful use of 125 microorganism-derived VOCs to control this disease.

126 Barleria prionitis (family Acanthaceae), known as an Ayurvedic herb, is endemic to tropical Asia and Africa (Sahu, 1984). It has been used as an herbal medicine to treat various health symptoms (Talukdar et al., 2015). Previous studies reported that this plant species contains several secondary metabolites that have antimicrobial activities (Amoo et al., 2011; Singh et al., 2005). It was hypothesized that this plant is a host of endophytic fungi that are capable of producing VOCs that suppress post-harvest fungal infections in fresh fruits. Therefore, this study aimed to isolate endophytic fungi from $B$. prionitis leaves and investigate the antifungal properties of their VOCs against $C$. acutatum mycelial growth and its infection on harvested strawberry fruits. The results of this study may lead to an alternative method to control post-harvest anthracnose disease caused by $C$. acutatum on fresh strawberry fruits.

136

\section{Materials \& Methods}

\section{Plant material}

Barleria prionitis plants, aged approximately 5 years, were collected from Chiang Rai province, Thailand in October 2019. Healthy B. prionitis leaves from the collected plants were sampled and sealed in plastic bags before being stored at $4{ }^{\circ} \mathrm{C}$ and used within $48 \mathrm{~h}$ of collection.

142

\section{Isolation of endophytic fungi}

The leaves of B. prionitis were washed with tap water to eliminate soil, dust, and dirt attached to the surface and rinsed with distilled water 3 times. The surface of the leaves was sterilized by immersing them in $70 \%$ ethanol for $1 \mathrm{~min}, 5 \%$ sodium hypochlorite for $30 \mathrm{sec}$, and $70 \%$ ethanol for $30 \mathrm{sec}$, to remove epiphytic microorganisms. After this, the leaves were washed thoroughly using sterilized distilled water to remove remaining solvents. The surface of the leaves was dried with sterilized tissue paper to provide aseptic conditions. The dried leaves were cut with a sterilized scalpel blade into small pieces (i.e., approximately $0.5 \mathrm{~cm} \times 0.5 \mathrm{~cm}$ segments). This surface sterilization procedure was performed following the study by Monggoot et al. (2017).

Each leaf segment was placed on a Petri plate containing potato dextrose agar (PDA) medium mixed with $30 \mu \mathrm{g} / \mathrm{mL}$ of chloramphenicol to prevent bacterial growth. A few drops of sterilized distilled water from the final washing step were placed on 5 PDA plates to confirm that no contamination was observed (negative controls). All PDA plates were sealed with Parafilm. The PDA plates were incubated at $27^{\circ} \mathrm{C}$ and examined daily for 7 days. Once the mycelia fully 
158

159

160

161

162

163

164

165

166

167

168

169

170

171

172

173

174

175

176

177

178

179

180

181

182

183

184

185

186

187

188

189

190

191

192

193

194

195

196

197

procedure was repeated at least 3 times until a pure culture of endophytic isolates was achieved. The endophytic isolates were coded as $\mathrm{BP}(\mathrm{x})$, where $\mathrm{x}$ was a numeric number assigned to each isolate.

\section{Isolation and molecular identification of phytopathogen $C$. acutatum}

Phytopathogenic fungus $C$. acutatum was isolated from 10 infected strawberry fruits that were collected from a strawberry field located at Angkhang Royal Agricultural Station in Fang district, Chiang Mai province, Thailand in February 2020. Each infected fruit was washed with sterile distilled water before transferring to a Petri plate containing PDA mixed with $30 \mu \mathrm{g} / \mathrm{mL}$ of chloramphenicol. They were incubated at $27^{\circ} \mathrm{C}$ for 7 days. All fungi obtained from the infected strawberry fruits were sub-cultured to obtain a pure culture. The isolated phytopathogen was preidentified based on micro-morphological and macro-morphological characteristics with sporulation. The aerial mycelium of $C$. acutatum isolate was scraped from the PDA surface and pulverized with a mortar and pestle to achieve a mycelium pulp. The genomic DNA was extracted following Tanapichatsakul et al. (2020). The internal transcribed spacer (ITS) was amplified using ITS5/ITS4 primers (Tanapichatsakul et al., 2020). Polymerase chain reactions (PCR) were performed following Tanapichatsakul et al. (2020). The sequence similarity of isolated fungi from BLAST result was $100 \%$ to C. acutatum. In addition, the pathogenicity test of an isolated pathogen was also performed using healthy strawberries to confirm pathogen identification. Ten strawberry fruits were wounded approximately $5 \mathrm{~mm}$ diameter and inoculated with a plug of C. acutatum. Fruits were kept in a moist plastic box and incubated at $27{ }^{\circ} \mathrm{C}$ for 7 days. Control fruits were inoculated with PDA agar plug. The test was performed in 3 replicates and repeated 3 times $(n=90)$. The symptoms and morphology of $C$. acutatum on strawberries were similar with previous studies (Kumvinit \& Akarapisan, 2016; Es-Soufi et al., 2018).

\section{In vitro screening of antifungal activity of VOCs produced by isolated endophytic fungi on the radial growth of $\boldsymbol{C}$. acutatum}

The VOCs produced by each isolated endophytic fungus against $C$. acutatum were screened for antifungal activity using the dual-culture plate method described by Li et al. (2015), with a slight modification on the incubation time. Two sterilized PDA bottom plates $(9 \mathrm{~cm}$ diameter $)$ were used. An agar plug ( $5 \mathrm{~mm}$ diameter, 7 days old) of $C$. acutatum was placed at the center of one plate, whereas an agar plug ( $5 \mathrm{~mm}$ diameter, 7 days old) of the individual endophytic isolate was placed at the center of another plate. The two plates were sealed together and wrapped with Parafilm before incubation at $27{ }^{\circ} \mathrm{C}$ for 7 days. Plates without the endophytic isolate but with C. acutatum were used as controls. The experiment was performed using 5 replicates for each endophytic isolate and repeated 3 times $(n=15)$. The mycelial diameter of $C$. acutatum in each treatment was measured daily using a vernier digital caliper (MITUTOYO-ABS Digimatic Caliper CD-AX, Japan) in a millimeter unit. The obtained results were expressed as inhibition percentage of mycelial growth according to the following formula: 
198

199

200

201

202

203

204

205

206

207

208

209

210

211

212

213

214

215

216

217

218

219

220

221

222

223

224

225

226

227

228

229

230

231

232

233

234

235

236

$\%$ Inhibition $=\left(\frac{\text { mycelial diameter of untreated fungus }- \text { mycelial diameter of treated fungus }}{\text { mycelial diameter of untreated fungus }}\right) \times 100$

\section{Viability test of the treated C. acutatum}

From the in vitro screening above, the treatment that exhibited the highest antifungal activity was selected for a viability test of the treated $C$. acutatum. An agar plug (5 mm diameter) of $C$. acutatum treated with the chosen endophytic isolate was placed on a fresh PDA plate and further cultured at $27^{\circ} \mathrm{C}$ for 7 days to evaluate its viability. The mycelial growth of C. acutatum was evaluated. The experiment was performed using 5 replicates and conducted 3 times $(n=15)$.

\section{In vivo antifungal activity of the VOCs produced by the endophytic isolate BP11 against $C$. acutatum infections on strawberry fruits}

The in vivo experiment was performed using healthy strawberry fruits collected in March 2020. All fruits were selected according to their maturity, size, color, and the absence of physical injuries or infections. The fruits were harvested early in the morning and then transported to the laboratory within 3 hours. The fruit surfaces were sanitized using 1\% sodium hypochlorite for 5 min, then rinsed with distilled water 3 times before being placed on a sterilized tissue paper for aseptic drying.

Inoculation of strawberry fruits with $C$. acutatum was performed according to the procedure described by Li et al. (2015), with a slight modification. One location on each cleaned strawberry fruit was wounded using a sterile inoculating needle. The wound depth was approximately $5 \mathrm{~mm}$. A plug of $C$. acutatum $(5 \mathrm{~mm}$ diameter, 7 days old) was placed into the wound. After inoculation, the strawberry samples were placed inside a plastic box $(15.5 \mathrm{~cm} \times 13.5$ $\mathrm{cm} \times 7.0 \mathrm{~cm}$ ). The bottom of the plastic box was covered with sterilized medical gauze. Double layers of autoclaved tissue paper soaked in $100 \mathrm{~mL}$ of sterile distilled water were placed underneath the gauze to provide moisture. Then, a 7-day old endophytic isolate BP11 culture plate, without its cover, was placed in the same box, but without direct contact with the strawberry samples. Each box was closed with a fitted plastic lid and air-sealed using Parafilm. All boxes were stored at $27^{\circ} \mathrm{C}$ for 7 days. To evaluate the effect of VOCs on the growth of C. acutatum, 4 different treatments were prepared, including pathogen-inoculated fruits in the plastic box (positive control), pathogen-uninoculated fruits in the endophytic isolate BP11-containing plastic box (negative control), pathogen-uninoculated fruits in the plastic box (control), and pathogeninoculated fruits in the endophytic isolate BP11-containing plastic box (BP11 treatment). There were 15 strawberry samples in each box. Each treatment was performed in 3 replicates and repeated 3 times $(n=135)$. The mycelial diameter of $C$. acutatum on strawberries from all treatments was measured using a vernier digital caliper in a millimeter unit. In this experiment, the inhibition percentage was calculated as explained above. Disease incidence and disease severity percentages were calculated using the following formulas:

$\%$ Disease incidence $=\left(\frac{\text { number of disease fruits }}{\text { total number of fruits assessed }}\right) \times 100$ 
$\%$ Disease severity $=\left(\frac{\text { sum of all disease rating }}{\text { total number of rating } \times \text { maximum disease scale }}\right) \times 100$

238

239

240

241

242

243

244

245

246

247

248

249

250

251

252

253

254

255

256

257

258

259

260

261

262

263

264

265

266

267

268

269

270

271

272

273

274

275

276

For the disease severity percentage, the rotten area on the surface of each strawberry fruit was visually evaluated using a scale from 0 to 5 . A description for each scale is as follows: $0=$ no disease symptom, $1=$ a small grey spot covering $<1 \%$ fruit area, $2=$ a grey sunken spot covering $1-10 \%$ fruit area, $3=$ a grey spot covering $11-25 \%$ fruit area, $4=$ circular grey sunken spots covering $26-50 \%$ fruit area, and $5=$ circular to irregular spots covering $>51 \%$ fruit area.

\section{Quality of strawberry fruits after treating with VOCs produced by the chosen endophytic isolate (BP11)}

The quality of strawberry fruits was evaluated after exposure to VOCs produced by the endophytic isolate BP11 for 7 days. In this experiment, fruit firmness, total soluble solids, and $\mathrm{pH}$ were measured following the method of Cai et al. (2015). Fruit firmness was measured by a TA-XT2i texture analyzer (Stable Micro Systems Ltd., UK) with a P50 cylinder plunger probe at the equator of the equidistant region. Fruit firmness was recorded from the maximum force. The texture analyzer was adjusted following this condition: $5.0 \mathrm{~mm} / \mathrm{s}$ pre-test speed, $1.0 \mathrm{~mm} / \mathrm{s}$ test speed, and $5.0 \mathrm{~mm} / \mathrm{s}$ post-test speed, all with a penetration distance of $5 \mathrm{~mm}$. Strawberry juice was extracted and filtered through two layers of cheesecloth. Total soluble solids of the strawberry fruit juice were measured using a hand-held refractometer (WYT-4) (Top Instrument Co., Ltd., China). The $\mathrm{pH}$ value was also determined by a pH meter (DELTA 320) (Top Instrument Co., Ltd., China). In this experiment, all treated strawberry samples from above were used.

\section{Identification and analysis of VOCs produced by the endophytic isolate BP11}

The VOCs produced by the endophytic isolate BP11 were extracted using solid phase microextraction (SPME). Prior to extraction, the endophytic isolate BP11 was cultured in a glass container $\left(9 \mathrm{~cm}\right.$ diameter) containing PDA medium at $27^{\circ} \mathrm{C}$ for 7 days and covered with an aluminum cap with a PTFE-coated silicone septum. The SPME sampling apparatus with a SPME holder and a $1.0 \mathrm{~cm}$ fused silica fiber was purchased from Supelco (PA, USA). A 50/30 $\mu \mathrm{m}$ divinylbenzene-carboxen-polydimethylsiloxane (DVB-CAR-PDMS) SPME fiber was selected to extract the volatile compounds. Before extracting the volatile compounds, the SPME fiber was preconditioned at $230^{\circ} \mathrm{C}$ inside the injection port of a gas chromatograph (HP 6890) (Agilent Technologies, CA, USA) for $30 \mathrm{~min}$. The preconditioned SPME fiber was then inserted into the glass container containing the endophytic isolate BP11 to trap the VOCs for 30 min prior to immersion in the injection port of the HP model 6890 gas chromatograph coupled to an HP model 5973 mass-selective detection instrument (Agilent Technologies) for $10 \mathrm{~min}$ in splitless mode. An HP-5ms ( $30 \mathrm{~m} \times 0.25 \mathrm{~mm}$ i.d., $0.25 \mu \mathrm{m}$ film thickness) column (Agilent Technologies) was used for compound separation. The oven temperature was initially set to $40{ }^{\circ} \mathrm{C}$ and then increased at a rate of $2{ }^{\circ} \mathrm{C} / \mathrm{min}$ to the final temperature of $200{ }^{\circ} \mathrm{C}$. The injector temperature was set to $250{ }^{\circ} \mathrm{C}$. Helium was used as a carrier gas and was set to a flow rate of $1 \mathrm{~mL} / \mathrm{min}$. The mass spectrometer was operated using electron impact ionization ( $70 \mathrm{eV}$ electron ionization voltages). A scan mode

Peer) reviewing PDF | (2020:10:53821:3:0:NEW 9 Mar 2021) 
277 (over the range of $\mathrm{m} / \mathrm{z} 30-300$ ) was used. The ion chamber and the mass transfer temperatures 278 were set to $250^{\circ} \mathrm{C}$ and $230{ }^{\circ} \mathrm{C}$, respectively.

279 The $\mathrm{C}_{9}-\mathrm{C}_{17} \mathrm{n}$-alkanes were used to calculate the retention time index. Mass spectra stored 280 in NIST05 Library, Wiley7N Mass Spectral Library, and Adams Library (2017) were used for

281

282

283

284

285

286

287

288

289

290

291

292

293

294

295

296

297

298

299

300

301

302

303

304

305

306

307

308

309

310

311

312

313

314

315

316 compound identification. A gas chromatograph (HP 6890, Agilent Technologies) equipped with a flame ionization detector was used for quantitative analysis of the volatile compounds. The identified volatile constituents were reported as the percentage of relative peak areas calculated using the normalization method without correction factors.

\section{In vitro antifungal activity of synthetic VOCs on C. acutatum}

Synthetic volatile standards of 3,5-dimethyl-4-heptanone (95\%), ethyl sorbate (95\%), benzaldehyde dimethyl acetal (95\%), trans-sabinene hydrate (95\%), methyl geranate (90\%), and elemicin (95\%) were used in this experiment. Ethyl sorbate, benzaldehyde dimethyl acetal, methyl geranate, and elemicin were purchased from Santa Cruz Biotechnology (TX, USA) while transsabinene hydrate was purchased from Sigma-Aldrich (China). These volatile standards were in a liquid form. The antifungal activity of each synthetic volatile compound was investigated according to the method of Gotor-Vila et al. (2017), with a slight modification. In this experiment, 2 sterilized PDA bottom plates (9 $\mathrm{cm}$ diameter) were used. An agar plug $(5 \mathrm{~mm}$ diameter, 7 days old) of C. acutatum was placed at the center of one plate, whereas a paper filter (90 $\mathrm{mm}$ diameter) spiked with an aliquot of the volatile standard was placed at the center of another plate. Both plates were sealed together and wrapped with Parafilm before incubation at $27{ }^{\circ} \mathrm{C}$ for 7 days. Each synthetic volatile compound was tested separately using 3 different aliquots (12.5, 25.0, and 50.0 $\mu \mathrm{L}$ ) of pure compounds. Because the Petri plates have a remaining headspace volume of $44 \mathrm{~mL}$, the corresponding concentrations of the synthetic compound were $0.28,0.56$, and $1.12 \mu \mathrm{L} / \mathrm{mL}$ headspace, respectively. Petri plates without synthetic VOCs but with C. acutatum were used as controls. For each synthetic volatile compound, 5 replicates for each spiking volume were used and repeated 3 times ( $\mathrm{n}=45$ for all 3 levels). The mycelial diameter of $C$. acutatum in each treatment was measured using a vernier digital caliper in a millimeter unit. The obtained results were expressed as inhibition percentage of mycelial growth as explained above.

\section{In vivo antifungal activity of elemicin against $C$. acutatum infections on strawberry fruits}

Strawberry fruits were prepared and wounded as explained above. They were placed at the bottom of the plastic box $(15.5 \mathrm{~cm} \times 13.5 \mathrm{~cm} \times 7.0 \mathrm{~cm})$ prepared in a similar manner as explained above. Then, a Petri plate containing 3 paper filters ( $90 \mathrm{~mm}$ diameter) moistened with $50 \mu \mathrm{L}$ of the elemicin standard was placed in the same box, but without direct contact with the strawberry samples. Each box was closed with a fitted plastic lid and air-sealed using Parafilm. All boxes were stored at $27^{\circ} \mathrm{C}$ for 7 days. Treatment that included only the pathogen-inoculated fruits in the plastic box was used as a positive control. In each box, there were 15 strawberry samples. Each treatment was performed in 3 replicates and repeated 3 times $(n=135)$. The mycelial diameter of 
317 C. acutatum on strawberry samples from all boxes was measured using a vernier digital caliper in 318 a millimeter unit. The inhibition, disease incidence, and disease severity percentages were 319 calculated as described above.

320

321

\section{Quality of strawberry fruits after treating with elemicin}

322 The quality of strawberry fruits was evaluated after treating with elemicin. This evaluation was done similarly to the procedure outlined above.

324

325

326

\section{Molecular identification of the endophytic isolate BP11}

Genomic DNA of endophytic isolate BP11 was extracted using a Genomic DNA Extraction Mini-

327

328

329

330

331

332

333

334

335

336

337

338

339

340

341

342

343

344

345

346

347

348

349

350

351

352

353

354

355 Kit following Tanapichatsakul et al. (2020). DNA loci including ITS, LSU, and RPB2 regions were amplified by PCR following the protocols of Samarakoon et al. (2019). The obtained sequences in this study were subjected to BLAST search in GenBank (https://blast.ncbi.nlm.nih.gov/Blast.cgi). BLAST search indicated that isolate BP11 belongs to Hypoxylaceae. The sequences of representative taxa in Hypoxylaceae used in the phylogenetic analyses were chosen from GenBank based on the BLASTn searches and recently published data (Samarakoon et al., 2019). Sequence datasets from all fungi obtained from ITS, LSU, and RPB2 rDNA primers were used to construct the phylogenetic tree. The accession numbers of all strains are shown in Table S1. The combined all sequence data were first aligned using MAFFT version 7 (http://mafft.cbrc.jp/alignment/server/). The alignment was further improved using BioEdit v. 7.0.5.3. The last alignment of the combined sequence datasets was investigated and the phylogenetic tree inferred based on maximum likelihood (ML) and Bayesian inference analyses (BI). ML and BI analyses were achieved using the RAxML-HPC2 on XSEDE (v. 8.2.12) via the CIPRES Science Gateway platform and MrBayes on XSEDE, MrBayes 3.2.6 via the CIPRES Science Gateway platform, respectively. Bayesian posterior probabilities (PP) were investigated using Markov Chain Monte Carlo sampling (BMCMC). The phylogenetic tree was constructed in FigTree v.1.4.3. The endophytic isolate BP11 was deposited in the Mae Fah Luang University Culture Collection (MFLUCC), Chiang Rai, Thailand under accession number MFLUCC 190493.

\section{Analysis of data}

Data from each experiment were presented as the mean \pm standard deviation. When applicable, data were subjected to Analysis of Variance (ANOVA), followed by post-hoc multiple pairwise comparisons using Duncan's multiple range tests $(\alpha=0.05)$ or Dunnett comparisons with a reference treatment $(\alpha=0.05)$. Linear regression analysis was conducted to evaluate the relationship between the observed inhibition percentage and the concentrations of synthetic VOCs tested. The statistical analyses were conducted using the SPSS 20.0 software (IBM Corp; 2011, NY, USA).

\section{Results}


356 357

358

359

360

361

362

363

364

365

366

367

368

369

370

371

372

373

374

375

376

377

378

379

380

381

382

383

384

385

386

387

388

389

390

391

392

393

394

In vitro antifungal activity of VOCs produced by endophytic isolates and viability of the treated C. acutatum

A total of 34 endophytic isolates were obtained from the B. prionitis leaves. After 7-day incubation, only 26 of them (Fig. S1) were able to produce VOCs that inhibit the mycelial growth of $C$. acutatum, but to varying degrees (Fig. 1). For these endophytic isolates, the inhibition percentages ranged between 3.3 and $80.8 \%$. The endophytic isolate BP11 was found to produce the VOCs with the highest inhibition percentage $(80.8, \mathrm{p}<0.05)$. Moreover, the in vitro antifungal activity of the volatile compounds produced by the endophytic isolate BP11 on C. acutatum across 7 days of the incubation period was depicted in Fig. S2. The highest antifungal activity was observed from day 4 to day 7. During which time, the average inhibition rate was $80 \%$. From the viability test, $C$. acutatum treated with the VOCs produced from the endophytic isolate BP11, could not reproduce its mycelia on the PDA plates over a 5-day incubation period (data not shown).

In vivo antifungal activity of VOCs produced by the endophytic isolate BP11 against $C$. acutatum infections on strawberry fruits

Strawberry samples after undergoing various treatments are shown in Fig. 2. Physical damage on the strawberry fruits was neither detected in the pathogen-uninoculated fruits containing the endophytic isolate BP11 (negative control) nor the pathogen-uninoculated fruits (control). In comparison to pathogen-inoculated fruits (positive control) (Fig. 2C), less physical damage was observed on fruits inoculated with C. acutatum and treated with the VOCs produced by the endophytic isolate BP11 (Fig. 2D).

\section{VOCs produced by the endophytic isolate BP11}

Gas chromatograph-mass spectrometric (GC-MS) analysis of the VOCs produced by the endophytic isolate BP11 led to the identification of 60 compounds. These VOCs are listed in Table 1. The volatile profile of the endophytic isolate BP11 was dominated by the presence of hydrocarbons $(35.0 \%)$, followed by monoterpene hydrocarbons $(39.4 \%)$, and sesquiterpene hydrocarbons (37.3\%). The major volatile compounds produced by the endophytic isolate BP11 were elemicin $(23.8 \%)$, benzaldehyde dimethyl acetal $(8.5 \%)$, ethyl sorbate $(6.8 \%)$, methyl geranate $(6.5 \%)$, trans-sabinene hydrate $(5.4 \%)$, and 3,5-dimethyl-4-heptanone $(5.1 \%)$. In addition, the VOCs produced from the endophytic isolate BP11 possessed sweet and fruity odor.

\section{In vitro antifungal activity of synthetic VOCs against $C$. acutatum}

The in vitro antifungal activities of the six most abundant VOCs against $C$. acutatum are summarized in Fig. 3. The results show that these chosen synthetic VOCs had varying degrees of antifungal activity. For every volatile compound tested, the highest inhibition percentage was detected on day 1 and continued to decrease in the later days $(p<0.05)$. Elemicin demonstrated the highest inhibition percentages across the observation period, covering a range of $15.3 \%$ to $76.6 \%$ $(\mathrm{p}<0.05)$ (Fig. 3). Ethyl sorbate showed poor antifungal activity against $C$. acutatum. 
395

396

397

398

399

400

401

402

403

404

405

406

407

408

409

410

411

412

413

414

415

416

417

418

419

420

421

422

423

424

425

426

427

428

429

430

431

432

433

434

Positive associations were found between the amounts of synthetic VOCs used (per mL headspace) and the inhibition percentages. In the case of elemicin, the resulting linear regression slope on day 1 of the incubation period was 22.1 (Fig. S3). Across the observation period, the slopes of elemicin were in a range of 22.1 to -24.7 . Moreover, the linear regression slopes of other compounds (on day 1) was also positive with a value of 22.5, 18.0, 13.4, 22.3, and 8.9 for 3,5dimethyl-4-heptanone, ethyl sorbate, benzaldehyde dimethyl acetal, trans-sabinene hydrate, and methyl geranate, respectively (data not shown).

\section{In vivo antifungal activity of elemicin against $C$. acutatum infections on strawberry fruits}

Strawberry fruits after treatment with elemicin are shown in Fig. 2E. Less physical damage was observed on the fruits inoculated with $C$. acutatum and treated with elemicin than the positive control samples. The inhibition, disease incidence, and disease severity percentages of this treatment were $70.9 \%, 21.5 \%$, and $15.9 \%$, respectively (Table 2 ). These results were similar to those obtained from the endophytic isolate BP11 treatment.

\section{Strawberry quality after treating with VOCs produced by the endophytic isolate BP11 or elemicin}

The quality of strawberry fruits obtained from various treatments, including the synthetic compound elemicin, in terms of firmness, total soluble solids, and $\mathrm{pH}$ are demonstrated in Table 3. After 7 days of treatment, the average firmness of pathogen-inoculated fruits (positive control) was $1.2 \mathrm{~N}$. It was significantly lower than other treatments. The average firmness of pathogeninoculated fruits (positive control), pathogen-uninoculated fruits containing the endophytic isolate BP11 (negative control), pathogen-uninoculated fruits (control), pathogen-inoculated fruits containing the endophytic isolate BP11 (BP11 treatment), and pathogen-inoculated fruits containing elemicin (elemicin treatment) ranged from 3.8 to $3.9 \mathrm{~N}$. These values were not significantly different. No significant difference was observed for the total soluble solids content (7.7-7.9) and the $\mathrm{pH}(3.3-3.4)$ of the strawberry fruits from all treatments.

\section{Phylogenetic tree and molecular identification}

The phylogenetic tree of the endophytic isolate BP11 is shown in Fig. 4. Based on the results, the endophytic isolate BP11 was identified as Daldinia eschscholtzii MFLUCC 19-0493. Its genetic sequence was submitted to the GenBank database under accession numbers MN704648, MW485486, and MW495050 from ITS, LSU, and RPB2 regions, respectively.

\section{Discussion}

The VOCs produced by the D. eschscholtzii MFLUCC 19-0493 possessed sweet and fruity odor, which is a feature of Daldinia fungi reported by Stadler et al. (2014). These VOCs were able to significantly inhibit the mycelial growth of $C$. acutatum without affecting the firmness, total 
435 soluble solids, and $\mathrm{pH}$ of the strawberry fruits, as shown in Table 3 . Thus, it is likely that 436 fumigation of harvested strawberry fruits with the VOCs produced from the D. eschscholtzii 437 MFLUCC 19-0493 will result in a prolonged storage time of harvested strawberry fruits by 438 reducing the incidence and severity of the anthracnose disease caused by $C$. acutatum. The 439 VOCs produced by the $D$. eschscholtzii MFLUCC 19-0493 is complex and comprised with at 440 least 60 compounds. In comparison to other major compounds identified, elemicin possessed the

441

442

443

444

445

446

447

448

449

450

451

452

453

454

455

456

457

458

459

460

461

462

463

464

465

466

467

468

469

470

471

472

473 strongest antifungal activity. The inhibition percentage of elemicin increased as the concentration increased (Fig. 3). This suggests that the ability for elemicin to suppress the mycelial growth of $C$. acutatum is dose-dependent. The same trend was observed for other synthetic VOCs tested.

The results of this study, regarding the antifungal activity of elemicin, agreed with previous studies, which also demonstrated that elemicin was a key antifungal volatile compound. In these studies, elemicin was found to be able to inhibit the growth of C. nymphaeae, C. gloeosporoides, and C. musae (Rossi et al., 2007; Karimi et al., 2016). In addition, other VOCs such as transsabinene hydrate (Btisam et al., 2016), ethyl sorbate (Nemes et al., 2020), and benzaldehyde dimethyl acetal (Goutam et al., 2016) were all reported to possess some degree of antimicrobial activity. The synergistic or additive actions of these compounds may increase the antifungal activity of the VOCs produced by the D. eschscholtzii MFLUCC 19-0493. The precise inhibitory mechanism of VOCc against $C$. acutatum needs to be elucidated through further study. To the best of our knowledge, this is the first time that the antifungal activity of elemicin was demonstrated against C. acutatum. According to the study of Tisserand and Young (2014), elemicin posed a negligible risk of carcinogenicity in humans at low doses. Thus, it has the potential to be used as a fumigant to control for C. acutatum infections on fresh strawberries. However, the production and ecological significance of elemicin from the D. eschscholtzii MFLUCC 19-0493 is not well understood and needs further investigation.

As observed from the in vitro screening experiment, the inhibitory patterns were different between the VOCs produced by the D. eschscholtzii MFLUCC 19-0493 and the selected synthetic volatile compounds. The observed inhibitory pattern of the synthetic VOCs was similar to the study of Gao et al. (2018) that the highest antifungal activity was present on the first day and decreased significantly on later days. The reduction in the inhibition percentage of the synthetic VOCs may be a result of a loss of chemical equilibrium or degradation from oxidation reaction. It is more likely that several volatile constituents contribute to the unique antifungal activity of the VOCs produced from D. eschscholtzii MFLUCC 19-0493. A few studies were able to propose the mechanisms by which certain VOCs inhibit the growth of pathogens. For instance, a combination of terpenoid compounds was found to inhibit the mycelial growth of selected pathogens by reducing the microbial cellular metabolic rates (Serrano et al., 2005; Regnier et al., 2010). Reduction of the metabolic rates may affect pathogenic microbial cell permeability and cell growth via reduction of the cell structure and its core function, causing the loss of macromolecules from the cell interior (Tian et al., 2012). In addition, the antifungal mechanisms of VOCs may depend 
474 on their ability to induce defense enzymes in the host rather than exerting the direct effect on the

475

476

477

478

479

480

481

482

483

484

485

486

487

488

489

490

491

492

493

494

495

496

497

498

499

500

501

502

503

504

505

506

507

508

509

510

511

512

pathogens, as suggested by Zhang et al. (2015).

Overall, the results of this study are in agreement with previous studies that reported the antifungal activity of the VOCs produced from Daldinia fungi. For instance, Liarzi et al. (2016) found that the VOCs produced by Daldinia cf. concentrica were able to control various plant pathogenic fungi. The major VOCs produced by D. cf. concentrica included trans-2-octenal, 1methyl-1,3-cyclohexadiene, 2,2,5-trimethylcyclopentanone, 1-methyl-1,4-cyclohexadiene, and octanal. However, the antifungal VOCs were 3-methyl-1-butanol, ( \pm )-2-methyl-1-butanol, 4heptanone, isoamyl acetate, and trans-2-octenal. The VOC profile obtained from $D$. cf. concentrica is substantially different from the one produced by D. eschscholtzii MFLUCC 19-0493. According to a previous study, some of the Daldinia fungi were reported to produce VOCs such as $\alpha$-guaiene, guaia-1(10),11-diene, (-)- $\alpha$-panasinsene, and thujopsene, which were identified as antimicrobial agents (Pažoutová et al., 2013). Also, the VOCs produced by Hyproxylon anthochroum, which is a member of the Daldinia genus, had strong antifungal activity against the growth of Fusarium oxysporum (Macías-Rubalcava et al., 2018). Moreover, 5-hydroxy-4,6-dimethyloctan-3-one isolated from Daldinia clavata as well as 5-hydroxy-2-methyl-4-chromanone isolated from Daldinia cf. childiae were evaluated to possess a high degree of antimicrobial activity (Wang et al., 2018; Lauterbach et al., 2019).

Due to the chosen extraction technique, some volatile compounds, such as small polyketides, which are mainly produced by the Daldinia fungi, could not be detected. These compounds are highly polar and were also reported to have antifungal activity (Liarzi et al., 2016). Our study is among just a few studies that have evaluated the use of endophytic fungal-derived VOCs as potential biofumigants to control for C. acutatum infections on strawberry fruits. A very recent study, conducted by Alijani et al. (2019), reported that the VOCs produced from the bacterial isolate Staphylococcus sciuri MarR44 possessed both in vitro and in vivo antifungal activities against $C$. nymphaeae, another pathogen that is responsible for anthracnose disease in fresh strawberry fruits. Our study is different from the study by Alijani et al. (2019) in terms of the selected pathogen and the target endophytic organism.

Our study suggests that VOCs from D. eschscholtzii MFLUCC 19-0493 could be used as fumigation agents to control the C. acutatum associated anthracnose disease by inhibiting the colonization of C. acutatum on fresh strawberry fruits during the post-harvest period. These VOCs, including the major compound, elemicin, are not phytotoxic to strawberry fruits. It is likely that small-scale farmers can use these VOCs to effectively control for this type of anthracnose disease. However, in order to utilize D. eschscholtzii MFLUCC 19-0493, there is a need to establish a sustainable condition to support the continued growth of the fungus. Further studies are needed to optimize the culture and to effectively maintain life-supporting conditions for $D$. eschscholtzii MFLUCC 19-0493 in order to enhance the antifungal efficiency of its producing VOCs. Determination of the optimum concentration of the VOCs produced by the D. eschscholtzii MFLUCC 19-0493 is also necessary for their effective use in controlling infections of C. acutatum

Peer) reviewing PDF | (2020:10:53821:3:0:NEW 9 Mar 2021) 
513 on strawberries during the post-harvest period. Lastly, more studies are needed to confirm the 514 effectiveness of elemicin against C. acutatum.

515

516 Conclusions

517 The present study showed that the VOCs produced by the D. eschscholtzii MFLUCC 19-0493

518 exhibited significant antifungal activity against the phytopathogen C. acutatum, which is usually 519 responsible for anthracnose disease on fresh strawberries, without causing changes in firmness, 520 total soluble solids, and $\mathrm{pH}$ of the fruits. Based on the GC-MS analysis, there were at least 60 521 VOCs produced by the D. eschscholtzii MFLUCC 19-0493. The major VOCs were elemicin, 522 benzaldehyde dimethyl acetal, trans-pinene hydrate, and 2-adamantanone. From the screening 523 assay, elemicin is likely the key VOCs that provides the majority of antifungal effect. Overall, the 524 volatile compounds produced by the D. eschscholtzii MFLUCC 19-0493 may be used as 525 biofumigation agents to control post-harvest infections of C. acutatum. The use of these VOCs for 526 fumigation of fresh strawberries is relatively inexpensive. They may be used by local farmers and 527 could replace synthetic chemicals or toxic gases that are currently used as fumigants to control the 528 post-harvest anthracnose disease caused by C. acutatum.

529

530

531

\section{Acknowledgements}

532

The authors would like to thank the Center of Excellence in Fungal Research, Mae Fah Luang 533 University for their support. We appreciate Dr. Sakon Monggoot for his support on the molecular analysis and fungal identification.

534

535

536

\section{Additional information and declarations}

537 This work was supported by the Mae Fah Luang University and the Royal Golden Jubilee Ph.D.

538 Programme for financial supporting through grant no. PHD/0193/2560.

539

540

\section{References}

541 Adams RP. 2017. Identification of essential oil components by gas chromatography/mass spectrometry, Allured Publishing Corporation, USA.

543

544

545

546

547

548

549

550

Alijani Z, Amini J, Ashengroph M, Bahramnejad B. 2019. Antifungal activity of volatile compounds produced by Staphylococcus sciuri strain MarR44 and its potential for the biocontrol of Colletotrichum nymphaeae, causal agent strawberry anthracnose. The International Journal of Food Microbiology 307:108276 DOI 10.1016/j.ijfoodmicro.2019.108276.

Amoo S, Ndhlala A, Finnie J, Van Staden J. 2011. Antifungal, acetylcholinesterase inhibition, antioxidant and phytochemical properties of three Barleria species. South African Journal of Botany 77(2):435-445 DOI 10.1016/j.sajb.2010.11.002. 
551

552

553

554

555

556

557

558

559

560

561

562

563

564

565

566

567

568

569

570

571

572

573

574

575

576

577

578

579

580

581

582

583

584

585

586

587

588

589

Arroyo F, Moreno J, Daza P, Boianova L, Romero F. 2007. Antifungal activity of strawberry fruit volatile compounds against Colletotrichum acutatum. Journal of Agricultural and Food Chemistry 55(14):5701-5707 DOI 10.1021/jf0703957.

Btisam O, Mohamed G, Badr S, Lahsen EG, Mariam F, Mounyr B, Elhassan EH, Benaissa K, Eddine BYS. 2016. Effect of harvest date on yield, chemical composition and antimicrobial activity of Artemisia herba-alba essential oil. International Journal of Scientific and Engineering Research 7(2):600-606.

Cai Z, Yang R, Xiao H, Qin X, Si L. 2015. Effect of preharvest application of Hanseniaspora uvarum on postharvest diseases in strawberries. Postharvest Biology and Technology 100:52-58 DOI 10.1016/j.postharvbio.2014.09.004.

Chalfoun NR, Castagnaro AP, Díaz Ricci JC. 2011. Induced resistance activated by a culture filtrate derived from an avirulent pathogen as a mechanism of biological control of anthracnose in strawberry. Biological Control 58(3):319-329 DOI 10.1016/j.biocontrol.2011.05.007.

Chen C, Cao Z, Li J, Tao C, Feng Y, Han Y. 2020. A novel endophytic strain of Lactobacillus plantarum CM-3 with antagonistic activity against Botrytis cinerea on strawberry fruit. Biological Control 148:104306 DOI 10.1016/j.biocontrol.2020.104306.

Es-Soufi R, L'bachir El Kbiach M, Errabii1 T, Saidi R, Badoc A, Chaveriat L, Martin P, Lamarti A. 2018. Biology and physiology of Colletotrichum acutatum strains causing strawberry's anthracnose. Agricultural Sciences 9:974-990 DOI 10.4236/as.2018.98068.

Giampieri F, Tulipani S, Alvarez-Suarez JM, Quiles JL, Mezzetti B, Battino M. 2012. The strawberry: composition, nutritional quality, and impact on human health. Nutrition 28(1):919 DOI 10.1016/j.nut.2011.08.009.

Gao H, Li P, Xu X, Zeng $Q$ and Guan W. 2018. Research on volatile organic compounds from Bacillus subtilis CF-3: Biocontrol effects on fruit fungal pathogens and dynamic changes during fermentation. Frontiers in Microbiology 9:456 DOI 10.3389/fmicb.2018.00456.

Gotor-Vila A, Teixidó N, Di Francesco A, Usall J, Ugolini L, Torres R, Mari M. 2017. Antifungal effect of volatile organic compounds produced by Bacillus amyloliquefaciens CPA-8 against fruit pathogen decays of cherry. Food Microbiology 64:219-225 DOI 10.1016/j.fm.2017.01.006.

Goutam J, Kharwar RN, Tiwari VK, Mishra A, Singh S. 2016. Isolation and identification of antibacterial compounds isolated from endophytic fungus Emericella qaudrilineata (RS-5). Natural Product Research 4(2): 1000205 DOI 10.4172/2329- 6836.1000205.

Huang R, Che HJ, Zhang J, Yang L, Jiang D, Li GQ. 2012. Evaluation of Sporidiobolus pararoseus strain YCXT3 as biocontrol agent of Botrytis cinerea on post-harvest strawberry fruits. Biological Control 62(1):53-63 DOI 10.1016/j.biocontrol.2012.02.010.

Kaddes A, Fauconnier ML, Sassi K, Nasraoui B, Jijakl MH. 2019. Endophytic fungal volatile compounds as solution for sustainable agriculture. Molecules 24(6):1065-1071 DOI $10.3390 /$ molecules 24061065

Peer) reviewing PDF | (2020:10:53821:3:0:NEW 9 Mar 2021) 
590 Kader AA. 1991. Quality and its maintenance in relation to the postharvest physiology of

591

592

593

594

595

596

597

598

599

600

601

602

603

604

605

606

607

608

609

610

611

612

613

614

615

616

617

618

619

620

621

622

623

624

625

626

627

628

629 strawberry. The strawberry into the 21st century. Timber Press, Portland, 145-152.

Karimi N, Salimikia I, Ramak P, Sohilikhah Z, Shamizadeh M, Gholivand M. 2016. Chemical composition, antioxidant and antimicrobial activities of essential oil from Leutea kurdistanica mozaff. Journal of Herbal Medicine 1(1):47-52.

Kuchi VS, Sharavani CSR. 2019. Fruit physiology and postharvest management of strawberry. IntechOpen DOI 10.5772/intechopen.84205.

Kumvinit A, Akarapisan A. 2016. Identification of Colletotrichum acutatum and screening of antagonistic bacteria isolated from strawberry in Chiang Mai, Thailand. International Journal of Agricultural Technology 12(4):693-706.

Lauterbach L, Wang T, Stadler M, Dickschat J. 2019. Volatiles from the ascomycete Daldinia cf. childiae (Hypoxylaceae), originating from China. Medicinal Chemistry Communications 10(5):726-734.

Li Q, Wu L, Hao J, Luo L, Cao Y, Li J. 2015. Biofumigation on post-harvest diseases of fruits using a new volatile-producing fungus of Ceratocystis fimbriata. PloS ONE 10:e0132009 DOI 10.1371/journal.pone.0132009.

Liarzi O, Bar E, Lewinsohn E, Ezra D. 2016. Use of the Endophytic fungus Daldinia $c f$. concentrica and its volatiles as bio-control agents. PLOS ONE 11(12):e0168242. DOI 10.1371/journal.pone.0168242.

Liu YB, Yang X, Simmons G. 2016. Efficacy of nitric oxide fumigation for controlling codling moth in apples. Insects 7(4):71 DOI 10.3390/insects7040071.

Macías-Rubalcava ML, Sánchez-Fernández RE, Roque-Flores G, Lappe-Oliveras P, Medina-Romero YM. 2018. Volatile organic compounds from Hypoxylon anthochroum endophytic strains as postharvest mycofumigation alternative for cherry tomatoes. Food Microbiology 76:363-373 DOI 10.1016/j.fm.2018.06.014.

Mercier J, Jiménez JI. 2004. Control of fungal decay of apples and peaches by the biofumigant fungus Muscodor albus. Postharvest Biology and Technology 31(1):1-8 DOI 10.1016/j.postharvbio.2003.08.004.

Monggoot S, Popluechai S, Gentekaki E, Pripdeevech P. 2017. Fungal endophytes: an alternative source for production of volatile compounds from agarwood oil of Aquilaria subintegra. Microbial Ecology 74(1):54-61 DOI 10.1007/s00248-016-0908-4.

Morath SU, Hung R, Ennett JW. 2012. Fungal volatile organic compounds: a review with emphasis on their biotechnological potential. Fungal Biolological Reviews 26(2-3):73-83 DOI 10.1016/j.fbr.2012.07.001.

Natsheh B, Abu Khalaf N, Mousa S. 2014. Strawberry (Fragaria ananassa Duch.) plant productivity quality in relation to soil depth and water requirements. International Journal of Plant Research 5(1):1-6 DOI 10.5923/j.plant.20150501.01.

Nemes D, Kovács R, Nagy F, Tóth Z, Herczegh P, Borbás A, Kelemen V, Pfliegler WD, Rebenku I, Hajdu PE, Fehér P, Ujhelyi Z, Fenyvesi F, Váradi J, Vecsernyés M, Bácskay I. 2020. Comparative biocompatibility and antimicrobial studies of sorbic acid derivates. 
630

631

632

633

634

635

636

637

638

639

640

641

642

643

644

645

646

647

648

649

650

651

652

653

654

655

656

657

658

659

660

661

662

663

664

665

666

667

668

669

European Journal of Pharmaceutical Sciences 143:105162 DOI 10.1016/j.ejps.2019.105162.

Pandey A, Banerjee D. (2014). Daldinia bambusicola $\mathrm{Ch} 4 / 11$ an endophytic fungus producing volatile organic compounds having antimicrobial and olio chemical potential. Journal of Advances in Microbiology 1(6):330 - 337.

Pažoutová S, Follert S, Bitzer J, Keck M, Surup F, Šrůtka P, Holuša J, Stadler, M. 2013. A new endophytic insect-associated Daldinia species, recognised from a comparison of secondary metabolite profiles and molecular phylogeny. Fungal Diversity 60(1):107123 DOI 10.1007/s13225-013-0238-5.

Pipattanawong N. 2015. Strawberry production and the royal project foundation, Thailand. Journal of Developments in Sustainable Agriculture 10(1):15-18.

Regnier T, Combrinck S, Du Plooy W, Botha B. 2010. Evaluation of Lippia scaberrima essential oil and some pure terpenoid constituents as postharvest mycobiocides for avocado fruit. Postharvest Biology and Technology 57(3):176-182 DOI 10.1016/j.postharvbio.2010.03.010.

Riyaphan P, Pipattanawong N, Subhadrabandu S. 2004. Influence of different climatic conditions on growth and yield of strawberry plants in Thailand. Production technologies for low-chill temperate fruits 9:65.

Rossi PG, Bao L, Luciani A, Panighi J, Desjobert JM, Costa J, Casanova J, Bolla JM, Berti L. 2007. (E)-Methylisoeugenol and elemicin: antibacterial components of Daucus carota L. essential oil against Campylobacter jejuni. Journal of Agricultural and Food Chemistry 55(18): $7332-7336$ DOI 10.1021/jf070674u.

Sahu T. 1984. Less known uses of weeds as medicinal plants. Ancient Science of Life 3:245.

Samarakoon SMBC, Samarakoon MC, Aluthmuhandiram JVS, Wanasinghe DN, Chomnunti P. 2019. The first report of Daldinia eschscholtzii as an endophyte from leaves of Musa sp. (Musaceae) in Thailand. Asian Journal of Mycology 2(1):183-197 DOI 10.5943/ajom/2/1/9.

Serrano M, Martínez-Romero D, Castillo S, Guillen F, Valero D. 2005. The use of antifungal compounds improves the beneficial effect of map in sweet cherry storage. Innovative Food Science and Emerging Technologies 6(1):115-123 DOI 10.1016/j.ifset.2004.09.001.

Singh B, Chandan B, Prabhakar A, Taneja S, Singh J, Qazi G. 2005. Chemistry and hepatoprotective activity of an active fraction from Barleria prionitis Linn. in experimental animals. Phytotherapy Research 19(5):391-404 DOI 10.1002/ptr.1509.

Sistrunk W, Morris J. 1983. Strawberry quality: influence of cultural and environmental factors. In Evaluation of quality of fruits and vegetables. Springer, Dordrecht.

Stadler M, Lassøe T, Fournier J, Decock C, Schmieschek B, Tichy H-V, Peršoh D. 2014. A polyphasic taxonomy of Daldinia (Xylariaceae) ${ }^{1}$. Studies in Mycology 77:1-143 DOI $10.3114 / \operatorname{sim} 0016$.

Suwannarach N, Kumla J, Bussaban B, Nuangmek W, Matsui K, Lumyong S. 2013. Biofumigation with the endophytic fungus Nodulisporium spp. CMU-UPE34 to control

Peer) reviewing PDF | (2020:10:53821:3:0:NEW 9 Mar 2021) 
670

671

672

673

674

675

676

677

678

679

680

681

682

683

684

685

686

687

688

689

690

691

692

693

694

695

696

697

postharvest decay of citrus fruit. Crop Protection 45:63-70 DOI 10.1016/j.cropro.2012.11.015.

Talukdar SN, Rahman MB, Paul S. 2015. A Review on Barleria prionitis: Its pharmacognosy, phytochemicals and traditional use. Journal of the Academy of Marketing Science 4(4):1-13 DOI 10.9734/JAMPS/2015/20551.

Tanapichatsakul C, Pansanit A, Monggoot S, Brooks S, Prachya S, Kittakoop P, Panuwet P, Pripdeevech P. 2020. Antifungal activity of 8-methoxynaphthalen-1-ol isolated from the endophytic fungus Diatrype palmicola MFLUCC 17-0313 against the plant pathogenic fungus Athelia rolfsii on tomatoes. PeerJ 8:e9103 DOI 10.7717/peerj.9103.

Tian J, Ban X, Zeng H, He J, Chen Y, Wang Y. 2012. The mechanism of antifungal action of essential oil from dill (Anethum graveolens L.) on Aspergillus flavus. PLoS One 7(1):e30147 DOI 10.1371/journal.pone.0030147.

Tisserand R, Young R. 2014. Essential oil safety 2nd edition, A Guide for health care professionals, Churchill Livingstone, 165-186.

Valenzuela NL, Angel DN, Ortiz DT, Rosas RA, García CFO, Santos MO. 2015. Biological control of anthracnose by postharvest application of Trichoderma spp. on maradol papaya fruit. Biological Control 91:88-93 DOI 10.1016/j.biocontrol.2015.08.002.

Wan M, Li G, Zhang J, Jiang D, Huang HC. 2008. Effect of volatile substances of Streptomycesplatensis F-1 on control of plant fungal diseases. Biological Control 46(3):552559 DOI 10.1016/j.biocontrol.2008.05.015.

Wang T, Mohr KI, Stadler M and Dickschat JS. 2018. Volatiles from the tropical ascomycete Daldinia clavata (Hypoxylaceae, Xylariales). Beilstein Journal of Organic Chemistry 14(9):135-147 DOI 10.3762/bjoc.14.9.

Zhang X, Sun Y, Yang Q, Chen L, Li W, Zhang H. 2015. Control of postharvest black rot caused by Altemaria altemata in strawberries by the combination of Cryptococcus laurentii and Benzo-(1,2,3)-thiadiazole-7-carbothioic acid S-methyl ester. Biological Control 90:96101 DOI 10.1016/j.biocontrol.2015.05.018. 


\section{Table $\mathbf{1}$ (on next page)}

Identified volatile compounds from the endophytic fungus $D$. eschscholtzii MFLUCC 19-0493 analyzed by SPME-GC-MS.

Retention index was determined using the homologous series of $\mathrm{n}$-alkanes. The \% peak area was calculated using the normalization method without correction factors. 
1 Table 1 Identified volatile compounds from the endophytic fungus D. eschscholtzii MFLUCC 19-0493 analyzed by 2 SPME-GC-MS.

\begin{tabular}{|c|c|c|c|c|c|}
\hline Compound & $\begin{array}{c}\text { retention } \\
\text { index }\end{array}$ & $\%$ area & Compound & $\begin{array}{c}\text { retention } \\
\text { index }\end{array}$ & $\%$ area \\
\hline 3,5-dimethyl-4-heptanone & 973 & 5.1 & 2-adamantanone & 1310 & 1.0 \\
\hline octen-3-ol & 974 & 0.1 & methyl geranate & 1322 & 6.5 \\
\hline 2-octanone & 988 & 0.3 & anisyl formate & 1330 & 1.0 \\
\hline 2,4,5-trimethyl-thiazole & 994 & 1.5 & 4-hydroxy-benzenemethanol & 1335 & 0.2 \\
\hline 2-ethyl-3-methyl-pyrazine & 1002 & 1.1 & silphinene & 1345 & 0.4 \\
\hline iso-sylvestrene & 1007 & 3.0 & 9-decenoic acid & 1359 & 0.5 \\
\hline 2-acetyl-thiazole & 1014 & 3.4 & para-methyl anisate & 1371 & 1.4 \\
\hline sylvestrene & 1025 & 0.7 & thujic acid & 1382 & 1.0 \\
\hline cis-arbusculone & 1046 & 0.2 & alpha-duprezianene & 1387 & 1.1 \\
\hline meta-tolualdehyde & 1069 & 0.2 & alpha-thujaplicin & 1410 & 0.1 \\
\hline 2E,4E-hexadienol acetate & 1081 & 3.0 & ethyl-anthranilate & 1414 & 0.3 \\
\hline diethyl acetal-2Z-hexenal & 1085 & 3.1 & beta-duprezianene & 1422 & 0.2 \\
\hline 2-acetyl thiophene & 1086 & 0.7 & dictamnol & 1428 & 0.1 \\
\hline ethyl sorbate & 1092 & 6.8 & pinonic acid & 1440 & 0.3 \\
\hline methylthiopyrazine & 1093 & 0.6 & prezizaene & 1444 & 0.3 \\
\hline trans-sabinene hydrate & 1098 & 5.4 & alpha-acoradiene & 1464 & 0.5 \\
\hline benzaldehyde dimethyl acetal & 1109 & 8.5 & gamma-muurolene & 1478 & 0.3 \\
\hline 2-ethyl hexanoic acid & 1119 & 0.5 & isodaucene & 1500 & 0.2 \\
\hline trans-pinene hydrate & 1119 & 2.0 & alpha-thujaplicinol & 1509 & 0.7 \\
\hline alpha-campholenal & 1122 & 0.3 & trans-calamenene & 1521 & 0.2 \\
\hline stemone & 1124 & 0.2 & zonarene & 1528 & 0.4 \\
\hline Z-myroxide & 1131 & 0.5 & cis-calamenene & 1528 & 0.1 \\
\hline iso-3-thujanol & 1134 & 1.9 & elemicin & 1555 & 23.8 \\
\hline 2-acetyl-3-ethyl-pyrazine & 1156 & 0.3 & E-nerolidol & 1561 & 0.3 \\
\hline fragranol & 1214 & 0.6 & Z-asarone & 1616 & 0.5 \\
\hline nor-davanone & 1228 & 0.4 & epi-cedrol & 1618 & 0.1 \\
\hline benzene acetic acid ethyl ester & 1243 & 0.9 & 5-cedranone & 1628 & 0.1 \\
\hline Z-anethole & 1249 & 1.6 & cedr-8(15)-en-10-ol & 1650 & 0.1 \\
\hline methyl nerolate & 1280 & 1.8 & 5-iso-cedranol & 1672 & 0.2 \\
\hline 2E,6Z-nonadienol acetate & 1303 & 0.6 & 5-neo-cedranol & 1684 & 0.6 \\
\hline
\end{tabular}

3

4 Retention index was determined using the homologous series of $n$-alkanes. The \% peak area was calculated using the 5 normalization method without correction factors. 


\section{Table 2 (on next page)}

Inhibition, severity, and incidence percentages of $C$. acutatum in strawberries treated with $D$. eschscholtzii MFLUCC 19-0493 and elemicin observed from the in vivo experiments.

The data are mean \pm standard deviation (total $n=135$ ) 
1 Table 2 Inhibition, severity, and incidence percentages of C. acutatum in strawberries treated with D. eschscholtzii

2 MFLUCC 19-0493 and elemicin observed from the in vivo experiments.

\begin{tabular}{lccc}
\hline Treatment & \% inhibition & \% severity & \% incidence \\
\hline D. eschscholtzii MFLUCC 19-0493 & $72.4 \pm 0.9$ & $19.9 \pm 0.8$ & $15.6 \pm 0.7$ \\
\hline elemicin & $70.9 \pm 1.2$ & $21.5 \pm 0.6$ & $15.9 \pm 0.9$ \\
\hline
\end{tabular}

3

$4 \quad$ The data are mean \pm standard deviation (total $n=135$ )

5 


\section{Table 3(on next page)}

Quality of fresh strawberries measured from the in vivo assays.

The data are mean \pm standard deviation ( $n=135$, per treatment). Different letters indicate significant differences $(p<0.05)$ among values within each category (ANOVA, followed by Duncan's multiple range test). 
$1 \quad$ Table 3 Quality of fresh strawberries measured from the in vivo assays

\begin{tabular}{|c|c|c|c|}
\hline \multirow{2}{*}{ Treatment } & \multicolumn{3}{|c|}{ Fruit quality } \\
\hline & Fruit firmness (N) & Total soluble solids (\%) & pH \\
\hline BP11 & $3.9 \pm 0.0^{\mathrm{a}}$ & $7.8 \pm 0.1^{a}$ & $3.3 \pm 0.0^{\mathrm{a}}$ \\
\hline Elemicin & $3.9 \pm 0.1^{a}$ & $7.7 \pm 0.1^{a}$ & $3.3 \pm 0.0^{\mathrm{a}}$ \\
\hline Control & $3.9 \pm 0.3^{a}$ & $7.9 \pm 0.0^{\mathrm{a}}$ & $3.3 \pm 0.0^{\mathrm{a}}$ \\
\hline negative control & $3.8 \pm 0.3^{\mathrm{a}}$ & $7.9 \pm 0.1^{\mathrm{a}}$ & $3.4 \pm 0.0^{\mathrm{a}}$ \\
\hline positive control & $1.2 \pm 0.8^{\mathrm{b}}$ & $7.7 \pm 0.1^{\mathrm{a}}$ & $3.4 \pm 0.0^{\mathrm{a}}$ \\
\hline
\end{tabular}

2

3 The data are mean \pm standard deviation ( $n=135$, per treatment). Different letters indicate significant differences 4 ( $p<0.05)$ among values within each category (ANOVA, followed by Duncan's multiple range test).

5

6 
Figure 1

In vitro antifungal screening of the endophytic isolates against the mycelial growth of $C$. acutatum (measured on day 7 of the incubation period).

The data for each bar are mean \pm standard deviation ( $n=15$ per isolate). Different letters above the bars indicate significant differences $(p<0.05)$ among the observed inhibition percentages (ANOVA, followed by Duncan's multiple range test).

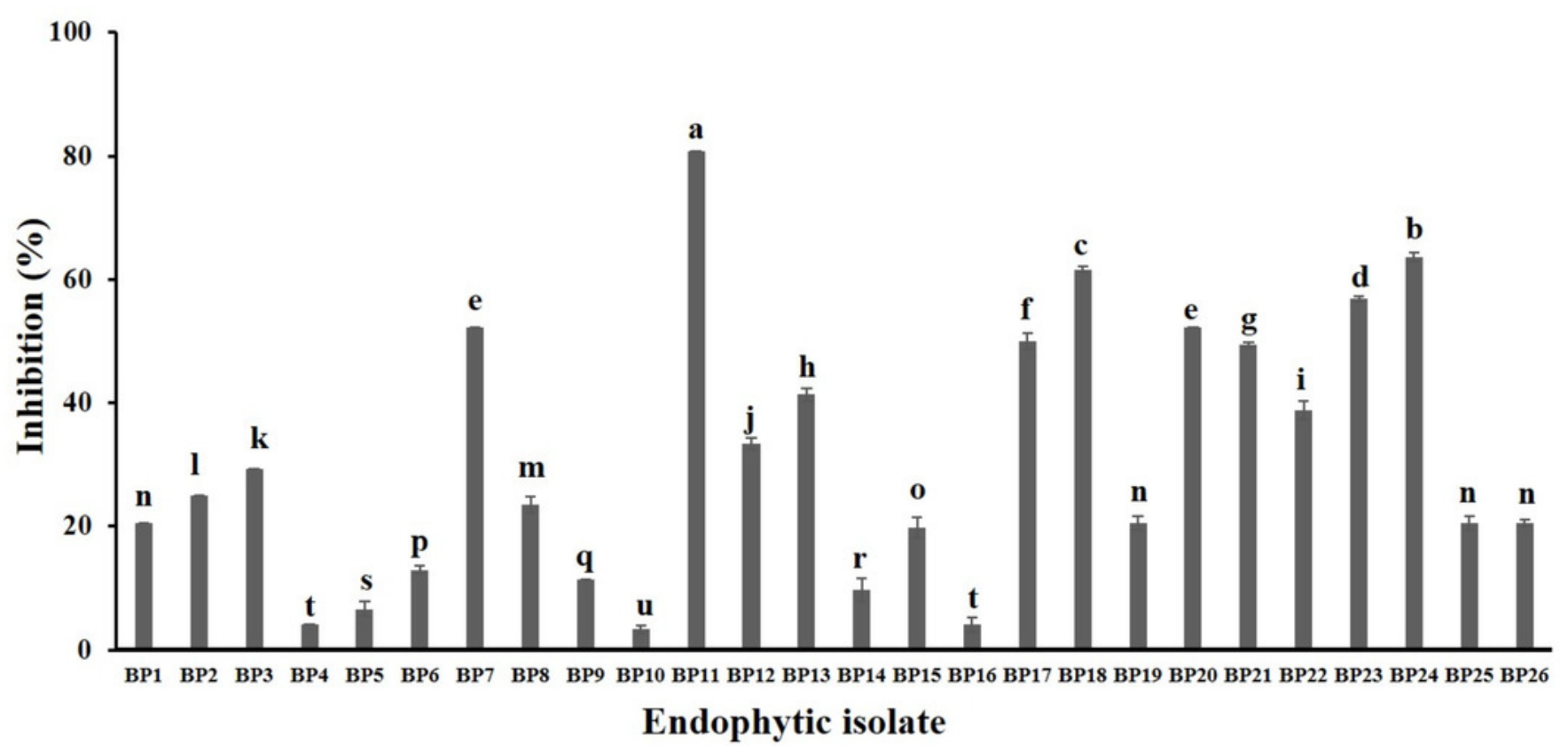


Figure 2

Examples of strawberries obtained from various in vivo experiments; negative control $(A)$, control (B), positive control (C), endophytic isolate BP11 (D), and elemicin (E).

Each treatment was performed in 3 replicates of $n=15$ and repeated 3 times (total $n=135$ ). 


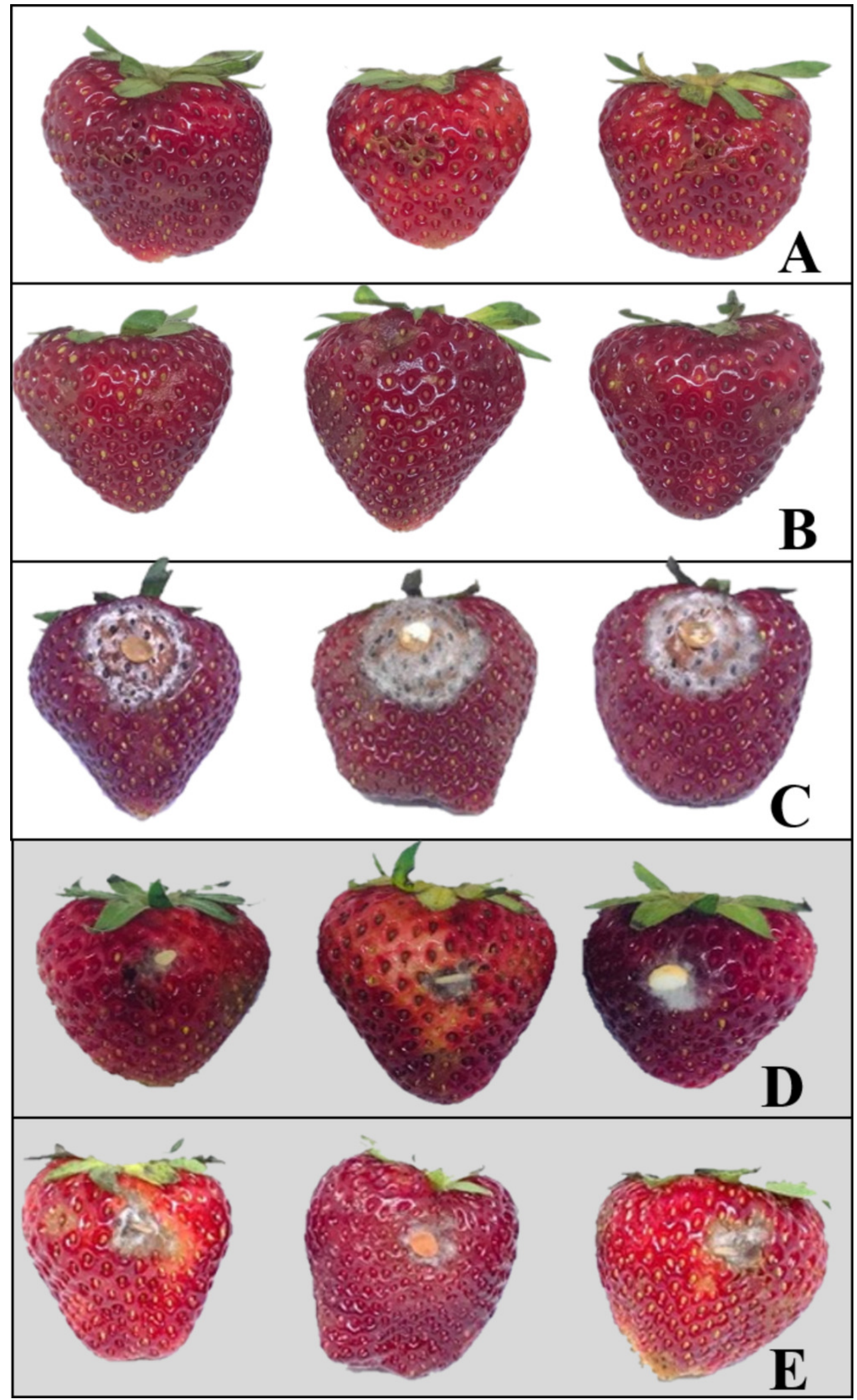

Peerj reviewing PDF | (2020:10:53821:3:0:NEW 9 Mar 2021) 


\section{Figure 3}

In vitro antifungal activity of synthetic volatile compounds at the starting concentration of 0.28 (A), 0.56 (B), and $1.12 \mu \mathrm{L} / \mathrm{mL}$ headspace (C) on C. acutatum during a 7-day incubation period.

The data for each point are mean \pm standard deviation ( $n=15$ per day). Sign $* * *$ above the points indicates a significant difference $(p<0.001)$ in the inhibition percentage between elemicin and other compounds observed in each day (ANOVA, followed by Dunnett's multiple range test). 

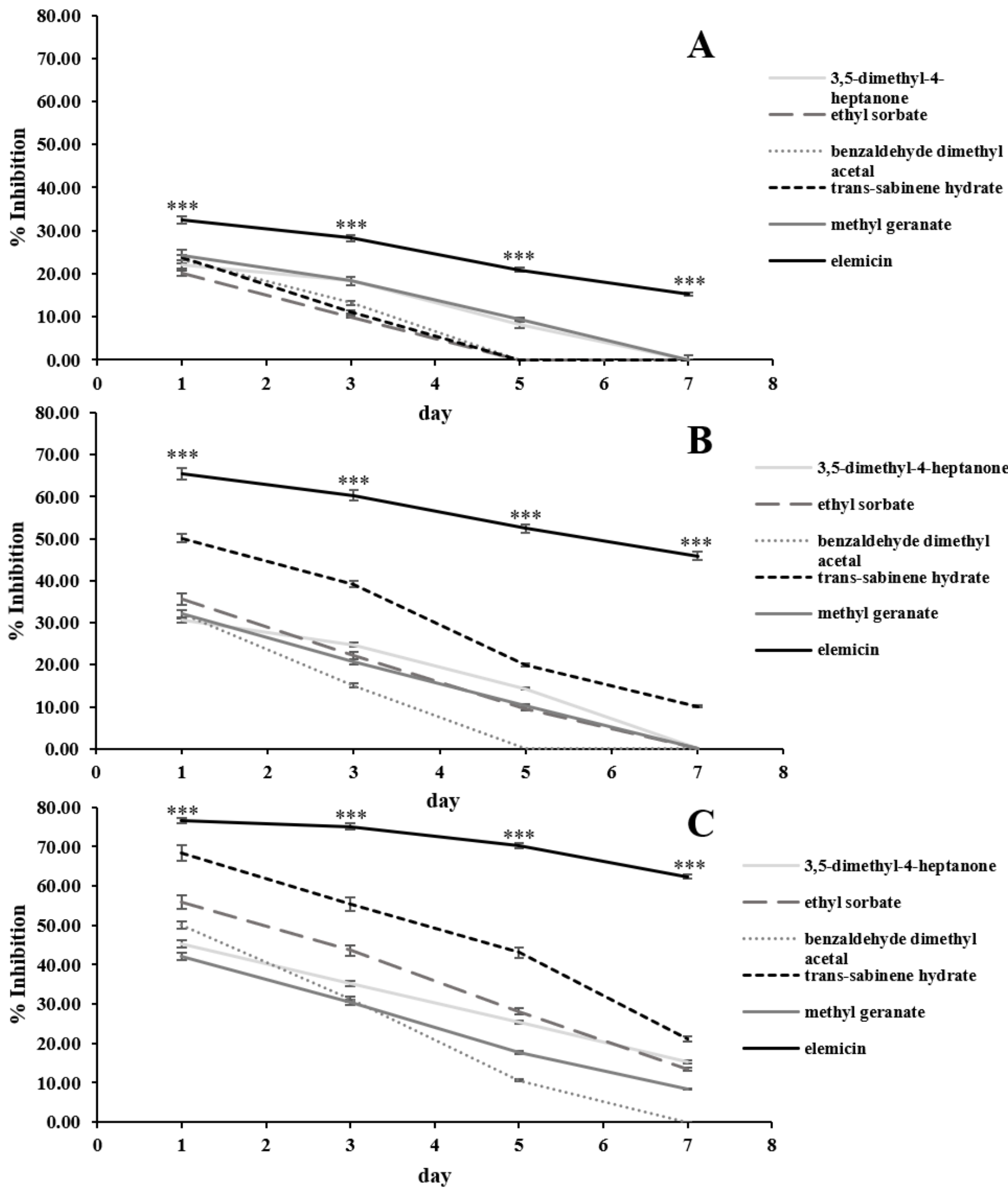
Figure 4

Phylogram generated from RAXML analysis based on a combined LSU-ITS-RPB2 rDNA sequence data of Daldinia, showing the phylogenetic position of $D$. eschscholtzii MFLUCC 19-0493.

Bootstrap support values for ML equal to or greater than $60 \%$, and Bayesian posterior probabilities (PP) equal to or greater than 0.90 are defined as ML/PP above the nodes. 


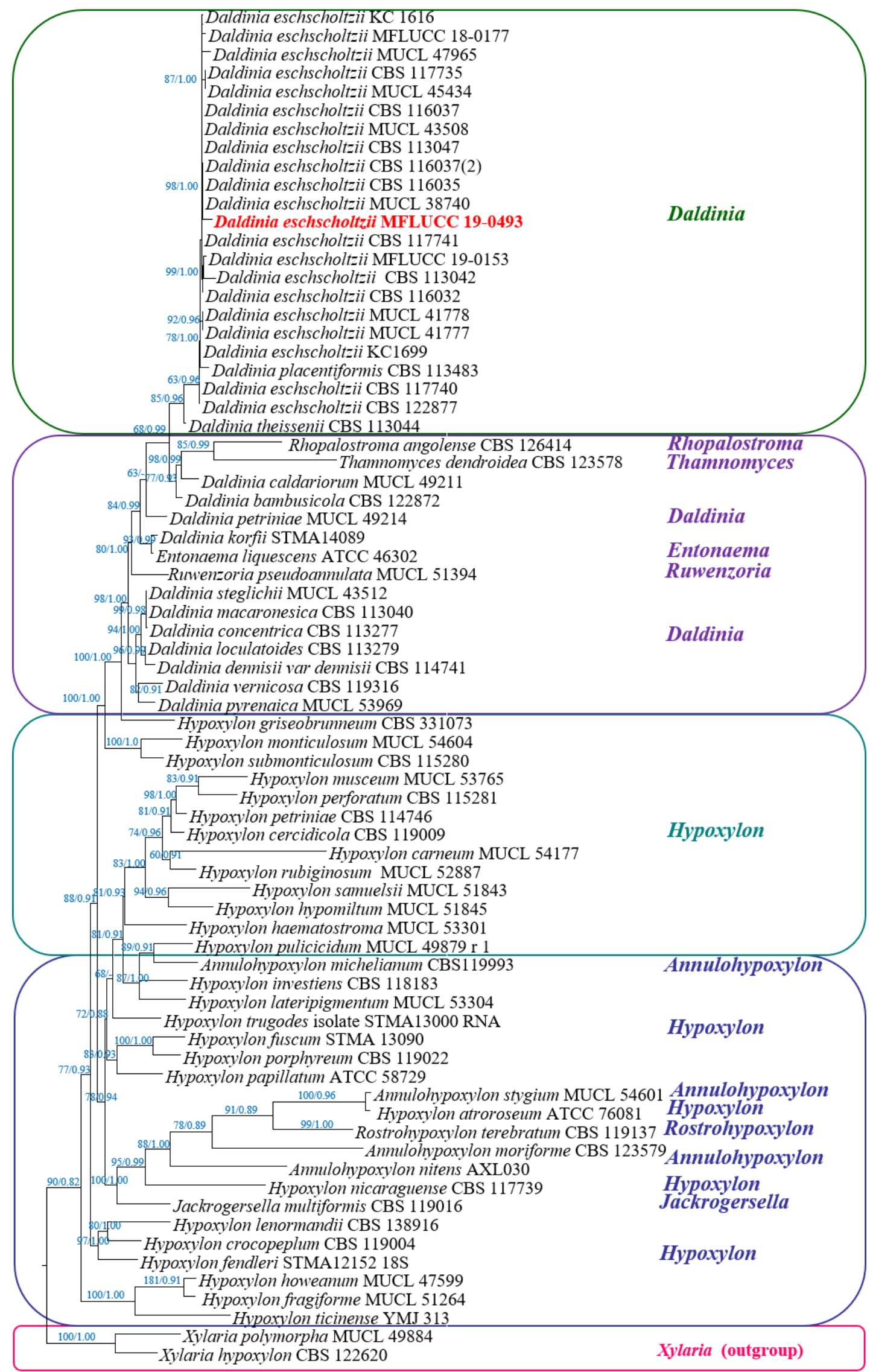

